\title{
Signature of local stress states in the deformation behavior of metallic glasses
}

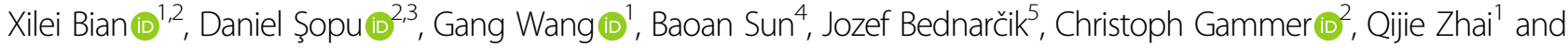 \\ Jürgen Eckert ${ }^{2,6}$
}

\begin{abstract}
The design of ductile heterogeneous metallic glasses (MGs) with enhanced deformability by purposely controlling the shear-band dynamics via modulation of the atomic-scale structures and local stress states remains a significant challenge. Here, we correlate the changes in the local atomic structure when cooling to cryogenic temperature with the observed improved shear stability. The enhanced atomic-level structural and elastic heterogeneities related to the nonaffine thermal contraction of the short-range order (SRO) and medium-range order (MRO) change the characteristics of the activation process of the shear transformation zones (STZs). The experimental observations corroborated by Eshelby inclusion analysis and molecular dynamics simulations disclose the correlation between the structural fluctuations and the change in the stress field around the STZ. The variations in the inclination axes of the STZs alter their percolation mechanism, affect the shear-band dynamics and kinetics, and consequently delay shear failure. These results expand the understanding of the correlation between the atomic-level structure and elementary plastic events in monolithic MGs and thereby pave the way for the design of new ductile metallic alloys.
\end{abstract}

\section{Introduction}

The plastic deformation of metallic glasses (MGs) at room temperature is inhomogeneous and highly localized into shear bands (SBs). Once SBs are triggered, such localized deformation and the related strain softening accelerate the instability of these SBs, eventually leading to catastrophic failure and essentially zero tensile plasticity $^{1,2}$. Controlling the SB dynamics and consequently improving the plastic deformability of MGs are challenging issues that are of crucial fundamental and practical importance. Tremendous efforts have been devoted to improving the room temperature ductility of MGs via modification of the glassy structure. The improvement in the mechanical properties has been widely attributed to an increase in the free volume (rejuvenation) ${ }^{3-5}$ or elastic

\footnotetext{
Correspondence: Gang Wang (g.wang@shu.edu.cn) or

Daniel Şopu (daniel.sopu@oeaw.ac.at)

'Laboratory for Microstructures, Institute of Materials, Shanghai University, 200444 Shanghai, China

${ }^{2}$ Erich Schmid Institute of Materials Science, Austrian Academy of Sciences, Jahnstraße 12, A-8700 Leoben, Austria

Full list of author information is available at the end of the article
}

fluctuations and structural heterogeneities (from atomicscale fluctuations to microscale crystalline inclusions) ${ }^{6-14}$. The underlying mechanisms are different: the former corresponds to lowered activation barriers for plastic events, while the latter is related to a broadened energy barrier distribution. While for composite materials, the interaction between SBs and crystalline inclusions has been used to explain the observed improvements in ductility $^{13,14}$, a thorough understanding of the intrinsic correlation between the atomic-scale structure and the macroscopic plasticity/ductility in monolithic MGs is still lacking, hindering the development of new high performance MGs. As a result, purposely designing the ductility by modulating the local atomic structure in MGs remains a major challenge.

The percolation of many shear transformation zones (STZs) along a maximum stress path remains the most plausible mechanism for SB formation. Following the pioneering work of Spaepen and Argon ${ }^{15,16}$, numerous simulations found that an STZ can be seen as a single plastic event in MGs characterized by a local stress field 
with quadrupolar symmetry ${ }^{17-19}$, reminiscent of the elastic field associated with the nucleation of a dislocation pair or the transformation of an Eshelby inclusion within an elastic continuum ${ }^{20}$. A quadrupolar-stress field symmetry is very similar to the basic symmetry of shear deformation in a disordered lattice of spherical-like atoms ${ }^{17}$. Although MGs lack long-range order, they still have a high degree of short-range order (SRO) and medium-range order (MRO), consisting of an interpenetrating network of close-packed and loose-packed atomic clusters ${ }^{15,16}$. Thus, although MGs are macroscopically homogeneous, the atomic-scale structure is highly heterogeneous, involving a distribution of atomic clusters with different effective bond strengths, which results in the formation of local soft spots enveloped by elastic surroundings ${ }^{21-23}$. Under loading, these soft spots show a high propensity for STZ activation ${ }^{21-23}$. Moreover, STZs behave as Eshelby inclusions ${ }^{17,24}$, and it has been predicted that an alignment of Eshelby-like quadrupoles in MGs can stimulate SB formation and plays an important role in the deformation behavior ${ }^{18}$. Moreover, STZs show complex antisymmetric stress fields, which in turn cause a vortex-like motion of the surrounding atoms. Such an autocatalytic STZ-vortex mechanism controls the percolation of STZs and, ultimately, SB dynamics and morphology ${ }^{25,26}$. However, once the structural state changes the stress field around the STZ, the axes of the activated STZs experience a large deviation from the maximum resolved stress affecting the STZ percolation path, which may further influence the shear-banding behavior $^{27}$. To date, the effects of the STZ activation and percolation processes associated with variations in the local stress state have not been elucidated and could have strong implications for the brittle-ductile transition in MGs.

To this end, by changing the environmental temperature, we attempt to modify the structural state to influence the activation of primary deformation units, such as STZs. In situ high-energy synchrotron X-ray diffraction (XRD) measurements are used to track the structural changes in real and reciprocal space. The relationship between the structural changes and the related stressstate modification is discussed in terms of stress field distributions around STZs, while an analytical model is proposed to explain the variations in the shear-banding angle. Finally, the atomic-scale origin of the observed variations in the $\mathrm{SB}$ dynamics and angle with changing structural state is revealed by molecular dynamic (MD) simulations.

\section{Materials and methods Tensile testing}

A $\mathrm{Zr}_{64.13} \mathrm{Cu}_{15.75} \mathrm{Ni}_{10.12} \mathrm{Al}_{10}$ (at\%) MG was chosen as the model material due to its excellent plasticity ${ }^{7}$. Alloy ingots were prepared by arc melting a mixture of high purity (>99.99\%) metallic elements, and the samples were remelted at least four times under a Ti-gettered argon atmosphere. Rod-like samples sized $3 \mathrm{~mm}$ (diameter) $\times$ $70 \mathrm{~mm}$ (length) were obtained by suction casting in a copper mold. Dog-bone-shaped tensile specimens with gauge dimensions of $2 \times 1 \times 1 \mathrm{~mm}^{3}$ (length $\times$ width $\times$ thickness) were machined from the as-cast rods and then finely polished. Uniaxial tensile tests were conducted using an MTS CMT5205 deformation machine with a temperature control facility at a strain rate of $5 \times 10^{-4} \mathrm{~s}^{-1}$. The strain measurement was obtained from the correction of the recorded displacement (from both the sample and testing machine). The test temperatures ranged from 293 to $77 \mathrm{~K}$. For each temperature, at least five specimens were tested. Prior to and during the tension tests, a cryogenic environment was attained and maintained at the preset temperatures by circulating liquid nitrogen through the specimen chamber. The fractography was examined using a scanning electron microscope (SEM, HITACHI SU-1500).

\section{In situ XRD measurements}

In situ XRD measurements were performed at the P02.1 beamline of the PETRA III electron storage ring (DESY Hamburg, Germany). The beam size was $0.6 \times 0.6 \mathrm{~mm}^{2}$, and the wavelength was $0.20727 \AA$. The sample $(1 \mathrm{~mm}$ diameter $\times 2 \mathrm{~mm}$ length) was held in a quartz capillary with a $1.2 \mathrm{~mm}$ diameter and $20 \mu \mathrm{m}$ wall thickness. The diffracted photons were collected using a 2D detector (Perkin Elmer PEI1621) mounted orthogonal to the X-ray beam. The sample-to-detector distance and tilting of the image plate detector with respect to the beam axis were calibrated using a diffraction pattern from LaB6 (NIST 660a). The samples were cooled from ambient temperature to $\sim 79 \mathrm{~K}$, followed by heating from $79 \mathrm{~K}$ up to $293 \mathrm{~K}$. The heating/cooling rates were $5 \mathrm{~K} / \mathrm{min}$. The obtained $2 \mathrm{D}$ diffraction patterns were integrated via Fit2D software ${ }^{28}$. From the integrated diffraction intensity, the structure factor $S(q)$ and the reduced pair-distribution function (PDF) $G(r)$ were calculated using the PDFgetX3 package $^{29}$. The peak positions of the structure factor $S(q)$ were extracted from a fit with a pseudo-Voigt function between $2.1 \leq q \leq 3.4 \AA^{-1}$.

\section{Molecular dynamics simulations}

The atomic-level mechanism of the shear banding was analyzed by MD simulations of a model $\mathrm{Cu}_{64} \mathrm{Zr}_{36}$ MG using the LAMMPS $\operatorname{code}^{30}$. No appropriate interatomic potentials exist for $\mathrm{Zr}_{64.13} \mathrm{Cu}_{15.75} \mathrm{Ni}_{10.12} \mathrm{Al}_{10}$ MG; hence, the $\mathrm{Cu}_{64} \mathrm{Zr}_{36}$ composition was chosen as a prototype because reliable Finnis-Sinclair type potentials are available $^{31}$. The simulated specimen was generated as follows. First, an MG cubic cell containing 8000 atoms was 
produced by quenching it from the melt to $50 \mathrm{~K}$ at a cooling rate of $10^{10} \mathrm{~K} / \mathrm{s}$. A softer glass was also prepared following the same procedure but using a higher cooling rate of $10^{12} \mathrm{~K} / \mathrm{s}$. Rectangular samples with dimensions of $46 \times 5 \times 10^{6} \mathrm{~nm}^{3}$ were then created by replicating the initial cell, resulting in a total of $\sim 1.5 \times 10^{6}$ atoms. These samples were termed hard and soft glasses, respectively. A small notch was introduced into the specimens to ensure that the plastic deformation was confined in a single SB, thus reproducing the experimental deformation behavior. The notched sample was loaded under uniaxial tension along the $z$-axis at $50 \mathrm{~K}$ using a strain rate of $4 \times 10^{7} \mathrm{~s}^{-1}$. The atomic von Mises stress/strain, rotation angle and rotation field were calculated and visualized using OVITO software. The local rotation $R$ was calculated by decomposing the atomic deformation gradient $F$ in the rigidbody rotation tensor and $U$ the right stretch tensor. In OVITO, the local rotation $R$ is encoded as a quaternion $R=(X, Y, Z, W)$. Thus, the rotation field was visualized by computing the angle of rotation (in radians) for each particle as $\theta=2 a \cos ($ Rotation. $W$ ) using the Compute Property modifier. In addition, the sign of the rotation angle was calculated using a Compute Property modifier as follows: $\operatorname{sign}($ Rotation. $Y) 2 a \cos ($ Rotation. $W$ ), where $Y$ is the direction perpendicular to the vortex (SB).

\section{Results}

\section{Tensile properties}

Typical tensile stress-strain curves of $\mathrm{Zr}_{64.13} \mathrm{Cu}_{15.75^{-}}$ $\mathrm{Ni}_{10.12} \mathrm{Al}_{10}$ MG at different temperatures are shown in Fig. 1a. Most monolithic MGs tensile tested at room temperature are prone to catastrophic fracture with zero plastic yielding after elastic deformation ${ }^{1,2}$. However, here, discernible strain to failure can be detected with decreasing temperature. Note that strain softening takes place once the maximum strength is reached, implying that inhomogeneous flow is the dominant method at cryogenic temperature $^{32}$. To better visualize this behavior, Fig. 1b shows a magnified view of the stress-strain curves indicated by the dashed rectangle in Fig. 1a, which clearly indicates that both the tensile strength and sustained strain to failure increase with decreasing temperature, as shown in the inset of Fig. 1b. The method for determining the yield strength and strain to failure is given in Supplementary Fig. 1. The tensile strength shows a linear relationship with temperature, and it increases by $\sim 350 \mathrm{MPa}$ when the temperature decreases from 293 to $77 \mathrm{~K}$. The strain to failure also increases with decreasing temperature, although it is improved marginally. Thus, it is obvious that decreasing temperature can yield higher strength and larger strain to failure in monolithic MGs.

\section{Structure evolution with decreasing temperature}

Usually, enhanced strength and ductility are attributed to structural changes ${ }^{33,34}$. However, the structural evolution due to changes in the environmental temperature is very insignificant. Here, high-energy synchrotron XRD investigations provide insights into the structural changes of MG with temperature. The structure factors in reciprocal space, $S(q)$, at different temperatures are plotted in Supplementary Fig. 2. The first maximum of $S(q)$ reflects the structural evolution in the MRO. The peak position of the first maximum shifts to a higher $q$ value with decreasing temperature, indicating a decrease in the mean atomic bond length. This corresponds to a volume contraction, which can influence the mechanical properties of the $\mathrm{MG}^{35}$. For example, the elastic modulus, $E$, of the MG monotonically increases with decreasing temperature (Supplementary Fig. 3), indicating continuous stiffening of the MG with decreasing temperature ${ }^{35}$. In addition, cooling from 293 to $79 \mathrm{~K}$ induces thermal stress, which is evaluated to be $\sim 10 \%$ of the macroscopic yield stress $^{3}$.
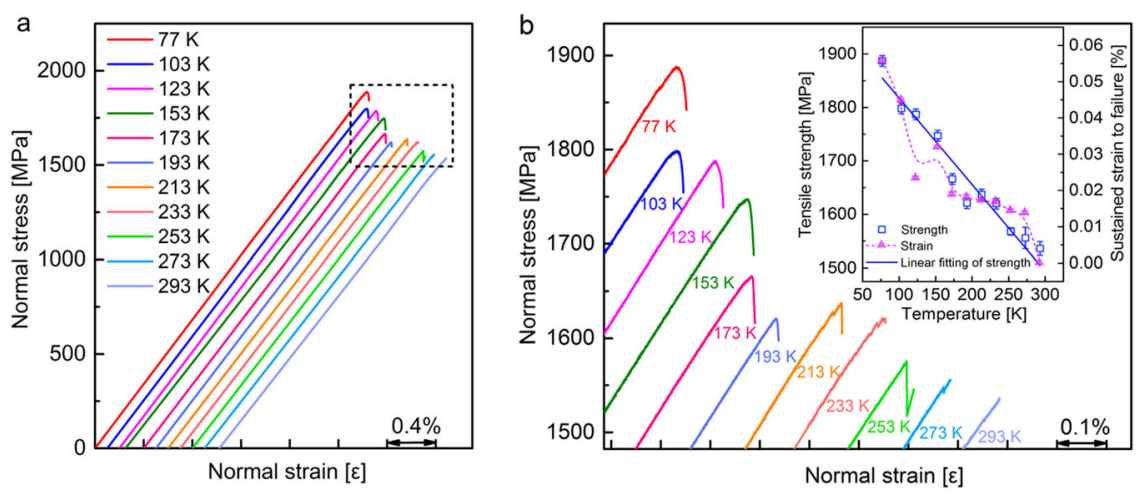

Fig. 1 Mechanical properties of $\mathrm{Zr}_{64.13} \mathrm{Cu}_{15.75} \mathrm{Ni}_{10.12} \mathrm{Al}_{10} \mathrm{MG}$ at different temperatures in the range of 77-293 K. a Representative tensile stress-strain curves. The starting points of the curves are set off for clearer viewing. $\mathbf{b}$ Magnified view of the tensile stress-strain curves marked by the dashed rectangle in (a). The tensile strength as well as the strain to failure as a function of temperature is shown in the inset. The lines are for guiding the eye. 

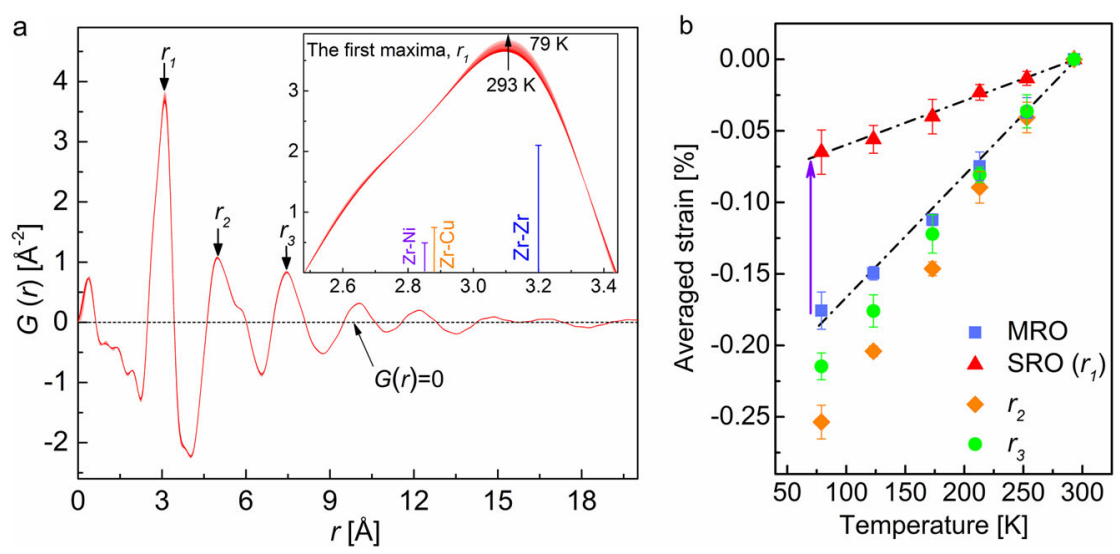

Fig. 2 Structural characterization of $\mathrm{Zr}_{64.13} \mathrm{Cu}_{15.75} \mathrm{Ni}_{10.12} \mathrm{Al}_{10}$ MG. a Representative reduced pair-distribution functions, $G(r)$, at different temperatures ranging from 293 to $79 \mathrm{~K}$. The inset shows the main three atomic pair correlations ( $\mathrm{Zr}-\mathrm{Zr}$, $\mathrm{Zr}-\mathrm{Cu}$, and $\mathrm{Zr}$-Ni) dominating the peak position shift in the first maximum. $\mathbf{b}$ Variations in the averaged strain calculated using the peak positions of $G(r)$ and $S(q)$ at different temperatures. Upon cooling, the MRO shows larger cooling contraction than the SRO, indicating that nonaffine thermal strain exists in the MRO and SRO. The lower the temperature is, the larger the difference in thermal strain. The fitted lines are for guiding the eye.

However, the evaluated thermal stress is much lower. The increase in the elastic modulus at $79 \mathrm{~K}$ is only $3 \%$ (Supplementary Fig. 3), which is not sufficient to explain the $\sim 23 \%$ increase in yield strength, implying that the yield strength increase not only depends on the affine contraction of the MRO (macroscopic contraction) underlying the cooling process but may also be linked to the changes in the SRO (degree of heterogeneity).

For the $\mathrm{Zr}$-based MG, the first maximum in the reduced PDF, $G(r)$, is a superposition of ten pair correlations (see Supplementary Table 1). Each partial pair correlation changes with temperature under the anharmonic and asymmetric nature of the interatomic interaction potential, leading to skewness of the non-Gaussian profile in the first maximum of $G(r)^{36-38}$, as shown in Fig. 2a. Therefore, caution should be taken in defining the position of the maximum for such an asymmetric and broad maximum. However, after some reasonable approximations, only three effective atomic pairs, i.e., $\mathrm{Zr}-\mathrm{Zr}, \mathrm{Zr}-\mathrm{Cu}$, and $\mathrm{Zr}-\mathrm{Ni}$, are considered ${ }^{39,40}$, as dominant contributions to $G(r)$ (see Fig. 2a). To explore the structural evolution of the SRO (the first coordination shell) and MRO (beyond the first coordination shell) in real space, the peak centerof-mass, $r_{c o m}$, of each coordination shell in $G(r)$ is determined by:

$$
r_{c o m}=\frac{\int_{r_{\min }}^{r_{\max }} G(r)}{\int_{r_{\min }}^{r_{\max }} G(r)}
$$

where $r_{\text {min }}$ and $r_{\text {max }}$ are the intersection coordinates of $G$ $(r)$ with the line $G(r)=0$. Based on the method developed by Poulsen et al. ${ }^{41}$ and further outlined by Shahabi et al. ${ }^{40}$, we can estimate the mean strain, $\varepsilon_{T}^{i}$, from the variations in the $G(r)$ peak position for the different coordination shells according to:

$$
\varepsilon_{T}^{i}=\frac{r_{T}^{i}-r_{293 \mathrm{~K}}^{i}}{r_{293 \mathrm{~K}}^{i}},
$$

where $r_{293 \mathrm{~K}}^{i}$ and $r_{T}^{i}$ represent the center-of-mass of the $i$-th shell in $G(r)$ at $293 \mathrm{~K}$ and $T$, respectively. Similarly, the mean strain can also be calculated by using the peak position of $S(q)$ in reciprocal space. From both real- and reciprocal-space analyses, the variations in the averaged strains for SRO and MRO as a function of temperature are shown in Fig. 2b, which clearly demonstrates that cooling contraction takes place for all coordination shells with decreasing temperature. However, this cooling contraction is nonuniform in different structure motifs (i.e., SRO and MRO), and the MRO contracts more, which is manifested by the wider difference in the mean strain (highlighted by arrow in Fig. 2b). The difference in the MRO on different length scales, such as the second and third coordination shells, becomes slightly stronger only at very low temperatures, indicating that nonaffine thermal strain primarily takes place in the MRO and SRO (Fig. 2b). That is, the SRO may experience cooling expansion due to local rejuvenation in addition to macroscopic cooling contraction when the temperature is down to the cryogenic level, which probably results from chemical SRO variations (changes in the $\mathrm{Zr}-\mathrm{Zr}, \mathrm{Zr}-(\mathrm{Cu}, \mathrm{Ni})$ atomic pairs $)$ in the first coordination shell during cooling ${ }^{34,42}$. Note that the averaged strains estimated in Fig. $2 b$ are inherently related to the structural density within and between the STZs. However, we must emphasize that the actual local variations may indeed be more pronounced than the magnitudes estimated here because the diffraction 
patterns are recorded over the entire interaction volume $\mathrm{e}^{11,23}$.

\section{Fracture angle and SB dynamics}

As demonstrated in Fig. 2b, a decrease in temperature leads to a nonaffine thermal strain in different structure motifs, rendering enhanced structural, and elastic heterogeneities $^{9-12}$. Although we cannot clearly distinguish between the local structural excitations and local elastic fluctuations, it is generally accepted that a higher degree of heterogeneity would change the characteristics of the STZ activation process and consequently affect the SB behavior, i.e., SB propagation direction and rate.

The SB propagation direction can be reflected by the shear fracture angle. The fracture angle, $\theta$ (or shearbanding angle), between the loading axis and the shear plane measured at different temperatures is shown in Fig. 3 (also see Supplementary Fig. 4). When the temperature decreases from 293 to $77 \mathrm{~K}$, the $\theta$ value increases from 54 to $60^{\circ}$, which is in excellent agreement with the theoretical prediction that all experimental $\theta$ values should fall in the range of $30-60^{\circ 43}$. Moreover, the $\theta$ value becomes larger with decreasing temperature, implying that the normal stress has a significant effect on the fracture angle ${ }^{43,44}$. This can be easily detected from the inset of Fig. 3, which clearly shows that the normal stress increases with decreasing temperature, while the shear stress remains almost constant. The fracture mode factor, $\omega$, i.e., $\omega=-\cot (2 \theta)^{43}$, characterizes the competition between the shear deformation and normal fracture under a uniaxial tension condition. The dependence of $\theta$ at different temperatures on $\omega$ is also plotted in Fig. 3, showing that the lower the temperature is, the larger $\omega$, which is consistent with the above analysis.

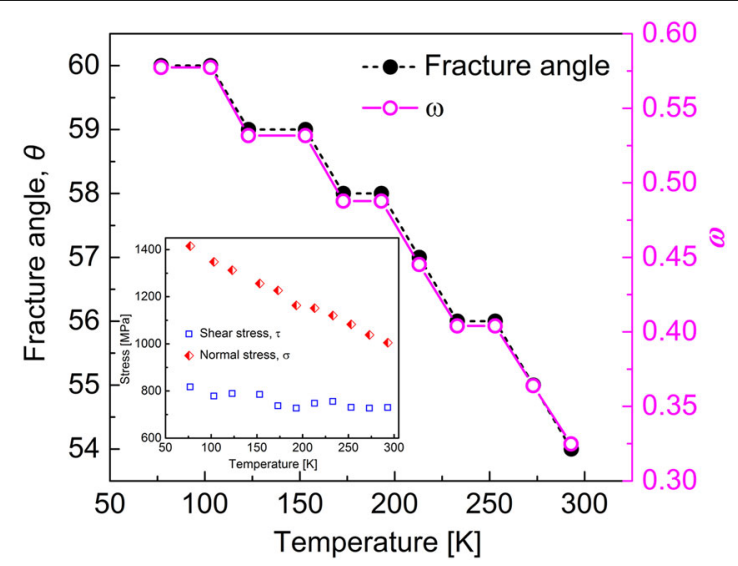

Fig. 3 Variations in the fracture angle $(\theta)$ and the fracture mode factor $(\omega)$ with decreasing temperature. The inset shows the shear and normal stresses on the failure plane as a function of temperature.
Based on the stick-slip shear dynamics and the atomicscale cooperative shearing model of STZs, the SB (inserting) sliding velocity, $v_{s}$, can be expressed as ${ }^{45}$ :

$$
v_{s}=v_{0} \exp \left[\frac{4 R \xi G_{0 T} \gamma_{C}^{2} \Omega}{k_{B} T}\left(1-\frac{\sigma}{\sigma_{0}}\right)^{3 / 2}+\frac{1}{\chi}\right],
$$

where $v_{0}$ is the external loading velocity, $k_{B}$ is the Boltzmann constant, $T$ is the temperature, $G_{0 T}$ is the shear modulus at different temperatures, $\gamma_{C}$ is the critical yield shear strain $\left(\gamma_{C} \approx 0.027\right), \Omega$ is the volume of the STZ, $R \approx 1 / 4, \xi \sim 2-4^{46}$, and $\sigma$ and $\sigma_{0}$ are the yield strengths at temperatures $T$ and $0 \mathrm{~K}$, respectively. $X$ is the effective temperature characterizing the state of the configuration disorder and the density or the total number of STZs ${ }^{47}$. The effective temperature is a dynamic quantity that depends on the strain rate and the temperature ${ }^{48}$ and evolves towards its kinetically ideal value, $\hat{x}$. Here, $\hat{X}=X_{w} / \ln \left(q_{c} / \dot{\gamma} t_{0}\right)^{49}$, where $q_{c}$ is the normalized strain rate at which the effective temperature diverges, $X_{w}$ is the normalized energy barrier that determines the effective temperature rate dependence, $\dot{\gamma}$ is the inelastic strain rate, and $t_{0}$ is the STZ time scale. An approximate analytical solution for the temperature dependence of $X$ is ${ }^{47}$ :

$$
\frac{1}{X}=\left\{\begin{array}{cc}
\frac{1}{\hat{\chi}} & T \leq T_{C} \\
\frac{1}{\hat{X}}-\frac{Q}{k_{B} \beta}\left(\frac{1}{T}-\frac{1}{T_{C}}\right) & T \geq T_{C}
\end{array},\right.
$$

where $Q$ is the activation energy for relaxation events, $\beta$ is the energy scale for the creation of relaxation events, and $T_{C}$ is the crossover temperature (173 K).

Substituting all the parameters into Eq. (3) allows evaluation of the SB sliding velocity, $v_{s}$, as a function of inverse temperature, as shown in Fig. 4. Apparently, the sliding velocity is much slower at cryogenic temperatures than at high temperatures and increases gradually below $173 \mathrm{~K}$, as indicated by the blue arrow in Fig. 4 . When the temperature is higher than $173 \mathrm{~K}$, the value of $v_{s}$ increases dramatically. It must be noted that the $v_{s}$ value below $173 \mathrm{~K}$ is comparable to that at room temperature under the compression mode ${ }^{50,51}$. Moreover, the $v_{s}$ value at room temperature can approach $1200 \mathrm{~m} / \mathrm{s}$, which is much faster than the speed of sound but slower than the Rayleigh wave speed, $v_{R}\left[v_{R} \approx 0.9225 c_{s}\right.$, where $c_{s}$ is the shearwave speed $(\sim 2100 \mathrm{~m} / \mathrm{s})]^{35,50}$. The $v_{s}$ value dramatically drops by approximately three orders of magnitude when the temperature is decreased from room temperature to the cryogenic level. Unlike the case of compression deformation, tensile deformation is usually dominated by a primary SB, which can have catastrophic consequences, resembling the fracture of brittle MGs with a high crack speed $^{52}$. 


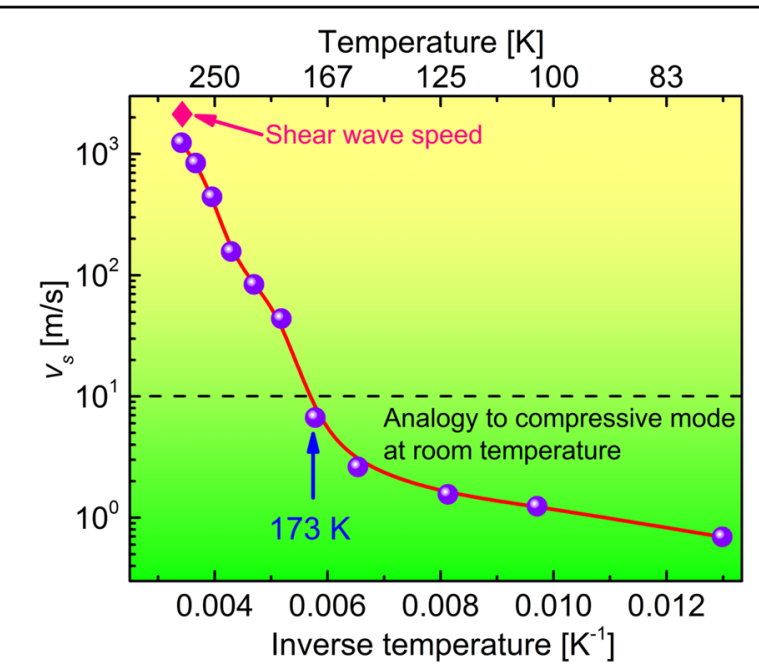

Fig. 4 Temperature dependence of the inserting SB sliding velocity $\boldsymbol{v}_{\boldsymbol{s}}$. The value of $\boldsymbol{V}_{\mathbf{s}}$ drops by approximately three orders of magnitude as the temperature decreases from 293 to $77 \mathrm{~K}$. The blue arrow indicates a transition point at $173 \mathrm{~K}$. The dashed line is to guide the eye, below which the value of $v_{s}$ is comparable to that at room temperature under compression mode.

With decreasing temperature, due to the cooling contraction, the overall density increases, while at the atomic scale, the degree of heterogeneity also increases due to the nonaffine thermal contraction. A global increase in density is equivalent to larger barriers for STZ activation, and consequently, reaching the macroscopic yielding point definitely requires more energy (higher loading stress), explaining the observed higher yield stress at low temperature (Fig. 1b). Moreover, microscopically, during plastic deformation, the shear-banding process is controlled by STZ percolation. While STZ activation requires higher energies (higher stresses), the percolation processes correlate to a high degree of heterogeneity due to the nonaffine thermal contraction in the SRO and MRO. In this case, the density of potential sites for SB nucleation should be enhanced in principle, and therefore, multiple SBs should form ${ }^{12,33}$. However, accommodating the imposed strain does not necessarily require activation of multiple SBs with decreasing temperature ${ }^{32}$. This is because the activation of a single SB (Supplementary Fig. 4) can also accommodate the imposed strain via strain softening behavior ${ }^{32}$, giving rise to a higher sustained strain to failure (see Fig. 1b). In addition, large variations in the structural/elastic heterogeneities perturb the percolation process, so the activation of a neighbor STZ becomes more difficult, which, consequently, leads to a slower STZ percolation rate and thus slower SB sliding velocity, and ultimately, runaway instability is retarded ${ }^{53}$. In other words, $10 \%$ of the increase in the yield strength relates to the affine contraction of the $\mathrm{MRO}$, while the rest (13\%) could be related to the enhanced degree of heterogeneity, which slows percolation of the STZs and delays SB formation and propagation. Therefore, these results suggest that the increased nonaffine thermal strain in the SRO and MRO may play a paramount important role in the STZ percolation path and influence the formation, morphology, and dynamics of the SB.

\section{Discussion}

Correlation between structure/stress state and stress field around the STZ

At this point, based on our experimental results, we can conclude that as the temperature decreases, an increasing difference in the nonaffine thermal strain occurs in different structure motifs (Fig. 2b), leading to enhanced structural heterogeneity, which in turn can promote the activation of more STZs. However, detailed characterization of the STZs in MGs is not straightforward because of the limited resolution of the measuring instruments. On the other hand, atomistic modeling and theory have shown that STZ activation results in long-range elasticdisplacement fields reminiscent of Eshelby inclusions ${ }^{17,20,24,25,27}$. Therefore, STZs behave as Eshelby inclusions experiencing shear strain in the elastic glassy matrix $^{15,16}$. The change in the local structure during the lowering of the temperature to the cryogenic level causes an additional thermal strain/stress in addition to shear strain due to the nonuniform cooling contraction (Fig. 2b). This, in turn, will have a strong effect on the STZ activation process, affecting the distribution of the strain and stress fields around the STZ due to the constraint of the glassy matrix (see Supplementary Note 1, Supplementary Table 2, and Supplementary Fig. 5).

An SB can be seen as a series of STZ-vortex arrangements $^{25}$. Here, we consider the response of one STZ under shear strain along the shear plane. Based on the stress field distributions displayed in Supplementary Fig. 5, the STZ percolation mechanism is schematically depicted in Fig. 5. When subjected to tensile stress, a quadrupolar-stress field will develop around the STZ. The tensile stress is oriented along the $y$-axis, whereas the compressive stress is perpendicular to it. The activated STZ can perturb the adjacent area by generating a strong antisymmetric-strain field that causes a collective vortexlike motion. The tensile strain compresses the material surrounding the vortex, and the compressive field generates traction below the vortex, as indicated by the green arrows in Fig. 5. The antisymmetric-strain field can drive the vortex to move, which then controls the activation of the following STZ. Thus, the repetition of such STZvortex arrangements leads to STZ percolation along a preferential direction with an angle of $\alpha_{1}$ and ultimately results in the formation and propagation of an SB when all STZs are activated and percolate along this $\alpha_{1}$-angle (see Fig. 5, left panel). When the temperature is decreased to 


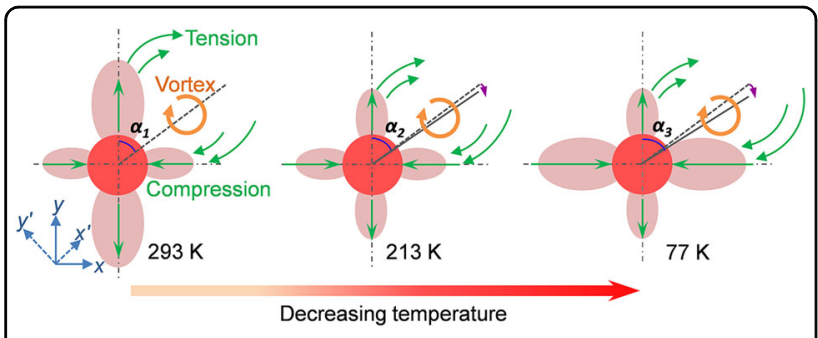

Fig. 5 Schematic illustration of the STZ (inclusion) percolation mechanism at different temperatures. Under tension, a quadrupolar-stress field is generated around an STZ, showing that the tensile stress (outwards pointing green arrows) acts along the $y$ direction and a compressive stress (inwards pointing green arrows) occurs perpendicular to it. The dashed lines show the angle of $a_{1}$ (percolation direction of the STZs), and the purple arrows indicate the deviation direction of the angle.

$213 \mathrm{~K}$, the stress field is similar to the case of $293 \mathrm{~K}$ (middle panel). However, the increased nonaffine thermal strain in the SRO and MRO perturbs the stress field around the STZ, and the compressive stress becomes larger than the tensile stress (see middle panel). Such a change in the stress distribution will govern the vortex alignment, which continuously deviates to the compressive stress. In this case, the activation of the next STZ-vortex sequence must deviate from the favorable orientation (along the $\alpha_{1}$-angle), and the SB propagation will be progressively hindered. The STZ must be aligned with the new local stress field, causing the $\mathrm{SB}$ to change direction towards a larger angle of $\alpha_{2}$ (middle panel). With a further decrease in the temperature to $77 \mathrm{~K}$, the compressive stress continues to increase, and the vortexformation site is closer to the direction of the compressive stress component. Hence, the angle of the STZ percolation path changes to a larger value of $\alpha_{3}$ than that at high temperature (see Fig. 5, right panel). Thus, we can easily deduce that $\alpha_{1}<\alpha_{2}<\alpha_{3}$ accounts for the observed inclination of the SB angle with decreasing temperature (Fig. 3 and Supplementary Fig. 4).

\section{Atomic-scale origin of the improved shear stability}

We demonstrated that the stress distribution around STZs dramatically changes with temperature, i.e., the compressive stress overwhelms the tensile stress, which perturbs the percolation of the STZs and changes the angle of the SB path with decreasing temperature down to the cryogenic level (Fig. 5). Moreover, the change in the magnitude of the stress around the STZs at $77 \mathrm{~K}$ will retard the generation of the adjacent vortex, and consequently, higher applied stresses are required to nucleate SBs and cause the glass to yield. Hence, the STZ-vortex sequence can be controlled by changing the environmental temperature, and thus, the SB dynamics can be influenced to delay runaway instability. The unexpected change in the stress state at cryogenic temperature is primarily attributed to the heterogeneous structure, with alternating soft and hard structure motifs resulting from the nonaffine thermal contraction in the SRO and MRO. Here, we must mention that structural and elastic variations within the glass as the temperature decreases to the cryogenic level will result in a higher degree of heterogeneity, consisting of soft spots with lower activation energy and hard spots with higher activation energy than the as-cast structure ${ }^{6-12,21-23}$. This is to say, a decrease in temperature leads to an increased nonaffine thermal strain. Moreover, the model proposed in Fig. 5 can be extended to explain the improved plasticity obtained by other approaches, such as thermal cycling ${ }^{3}$, triaxial compression $^{4}$, hyperquenching ${ }^{8}$, fatigue ${ }^{9}$, severe plastic deformation $^{12}$, and second crystalline phases ${ }^{13,14}$. Although all these methods will increase the structural heterogeneities in the structure and generate mostly soft/ rejuvenated regions, resulting in a lower macroscopic yield strength, our model can still explain the observed shear stability. To verify the importance of soft spots on the deformation and thus validate our model, we performed MD simulations on two kinds of structures, a soft and a hard MG obtained using different cooling rates, with the same chemical composition but different amounts (distributions) of free volume and degrees of SRO (Supplementary Fig. 6). The variations in the stress and strain at the atomistic level during SB formation are compared for the hard MG (with a small number of soft spots) and the soft MG (with a large number of soft spots). The reason for choosing simulations at $50 \mathrm{~K}$ with different cooling rates is detailed in Supplementary Note 2 and Supplementary Fig. 7.

The evolutions of the atomic von Mises strain and the rotation angle of the hard and soft MGs under loading are shown in Fig. 6. For both MGs, the strain distribution is highly discontinuous within the evolving SB, consisting of isolated regions (STZs, red circles) with larger strain separated by regions with strong rotation (vortexes, yellow circles) but smaller strain (Fig. 6). To better visualize the vortex motion, the time evolution during the deformation of a randomly chosen vortex based on displacement vectors is shown in Supplementary Movie 1. The alternating sequence of STZs and vortex elements is correlated with a systematic variation in the von Mises stress (dashed blue curves in Fig. 6), showing minima at the position of the STZs (rose colored region) and maxima at the position of the vortexes (orange colored region). The variation in the von Mises stress is in the range of $6 \times 10^{5}-10 \times 10^{5}$ atm $\AA^{3}$ for the hard MG, whereas it is between $2 \times 10^{5}$ and $8 \times 10^{5} \mathrm{~atm} \AA^{3}$ for the soft MG, showing a wider variation than the hard MG (dashed blue curves in Fig. 6). To quantitatively characterize this difference, we computed the stress difference 


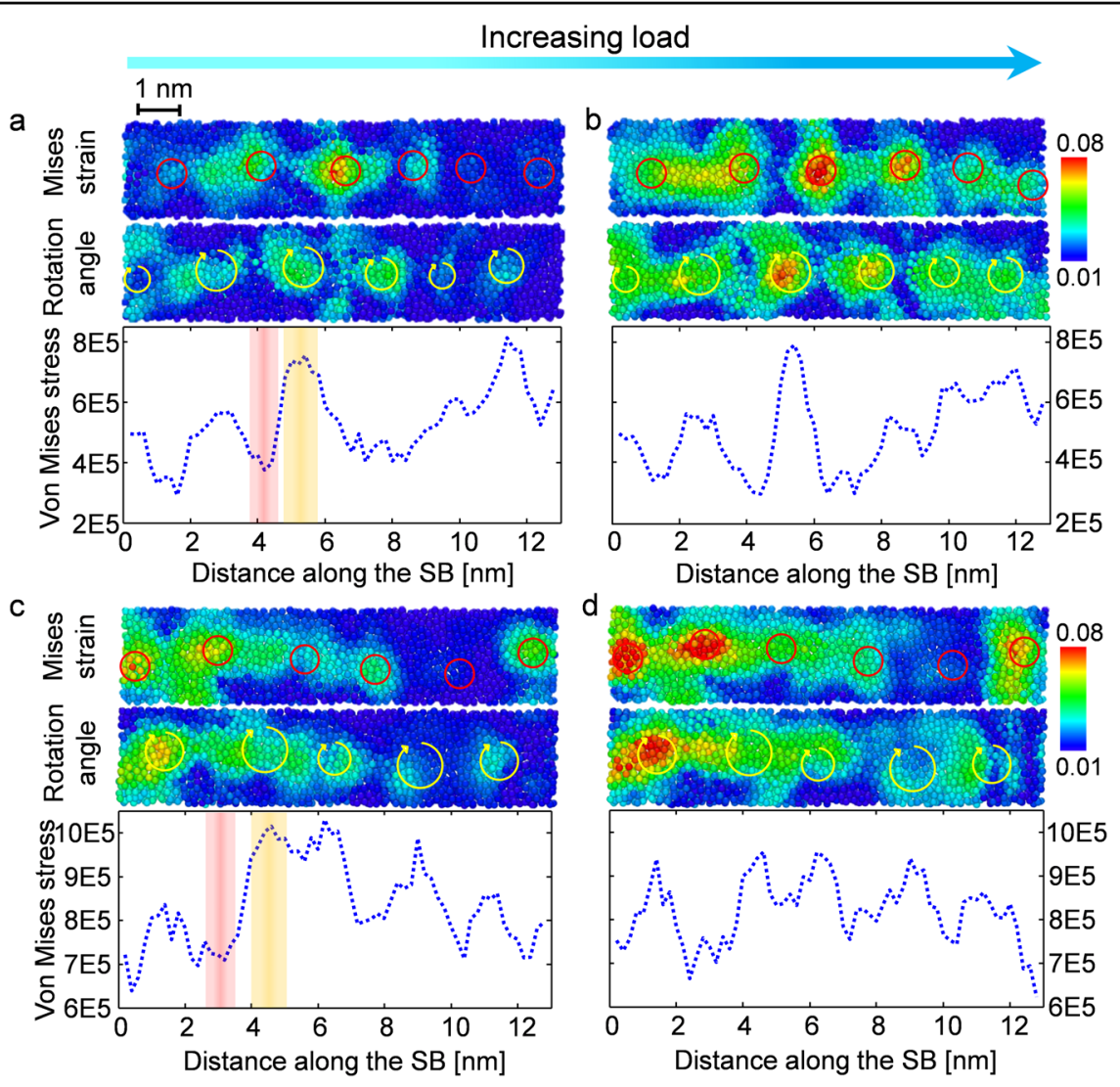

Fig. 6 Simulation files of the soft and hard samples. Evolutions of the atomic von Mises stress/strain and rotation angle along the SB of the simulated soft $[(\mathbf{a})$ and $(\mathbf{b})]$ and hard $[(\mathbf{c})$ and $(\mathbf{d})]$ samples under loading. For both samples, strong rotation and strain fields are mutually exclusive: areas with the largest rotation (vortexes, yellow circles) exhibit reduced von Mises strain (STZs, red circles), whereas strong strain coincides with smaller rotation. The corresponding von Mises stress (unit, atm $\times \AA^{3}$ ) curves also display a rather systematic variation, i.e., the stress is low at the STZ position (rose colored region) and relatively high at the vortexes (orange colored region).

between the positions of the STZs and vortexes. The average difference in the von Mises stress is higher for the soft MG than for the hard MG $\left(3.26 \times 10^{5}\right.$ atm $\AA^{3}$ and $2.21 \times 10^{5}$ atm $\AA^{3}$, respectively). Since shear banding is a stress-driven process ${ }^{25}$, the evolution of the shear front will be strongly affected by the local stress field. We can thus expect that the higher stress required to generate a vortex from an STZ perturbation in the soft MG is likely to slow down the continuum percolation of STZs and the formation of critical SBs.

Next, the correlation between the structural fluctuations and the change in the SB angle is investigated by using the sign of the rotation angle with the $y$-axis, as shown in Fig. 7. Since the rotation field shows a quadrupolar-like distribution around the STZs, it can be used as a diagnostic tool to highlight the axes of the activated $\mathrm{STZs}^{27}$. The rotation direction is clockwise (white color) along the shear front and counterclockwise (black color) perpendicular to it. The change in the stress distribution around the STZ directly causes variation in the inclination axes. Hence, the STZ percolation path and SB formation follow different specific directions, leading to SB propagation with a higher angle in the soft sample (see lower panels in Fig. 7). The increase in the shear angle for MGs with soft structures is also observed experimentally ${ }^{12}$ (see Fig. 3 and Supplementary Fig. 4). Moreover, the stress accumulation process of the soft sample must slow the rate of STZ percolation and thus prolong the time for SB formation, which ultimately decreases the sliding velocity of the inserting SB (see Fig. 4).

Soft heterogeneities, in composite materials, soft crystalline second phases ${ }^{13,14}$, or heterogeneities generated by thermal or mechanical treatments ${ }^{3,4,8-12}$ introduce strain concentrations, acting as initiation sites for shear banding. Such heterogeneities can be considered an effective means for stress-state modification that can enhance the deformability of MGs. The nanoscale MD simulations shown above demonstrate that our model proposed in Fig. 5 can effectively capture the underlying mechanism of the enhanced shear stability through the alteration of the stress-state distribution via nanoscale structural heterogeneities. The connection between the simulation and 


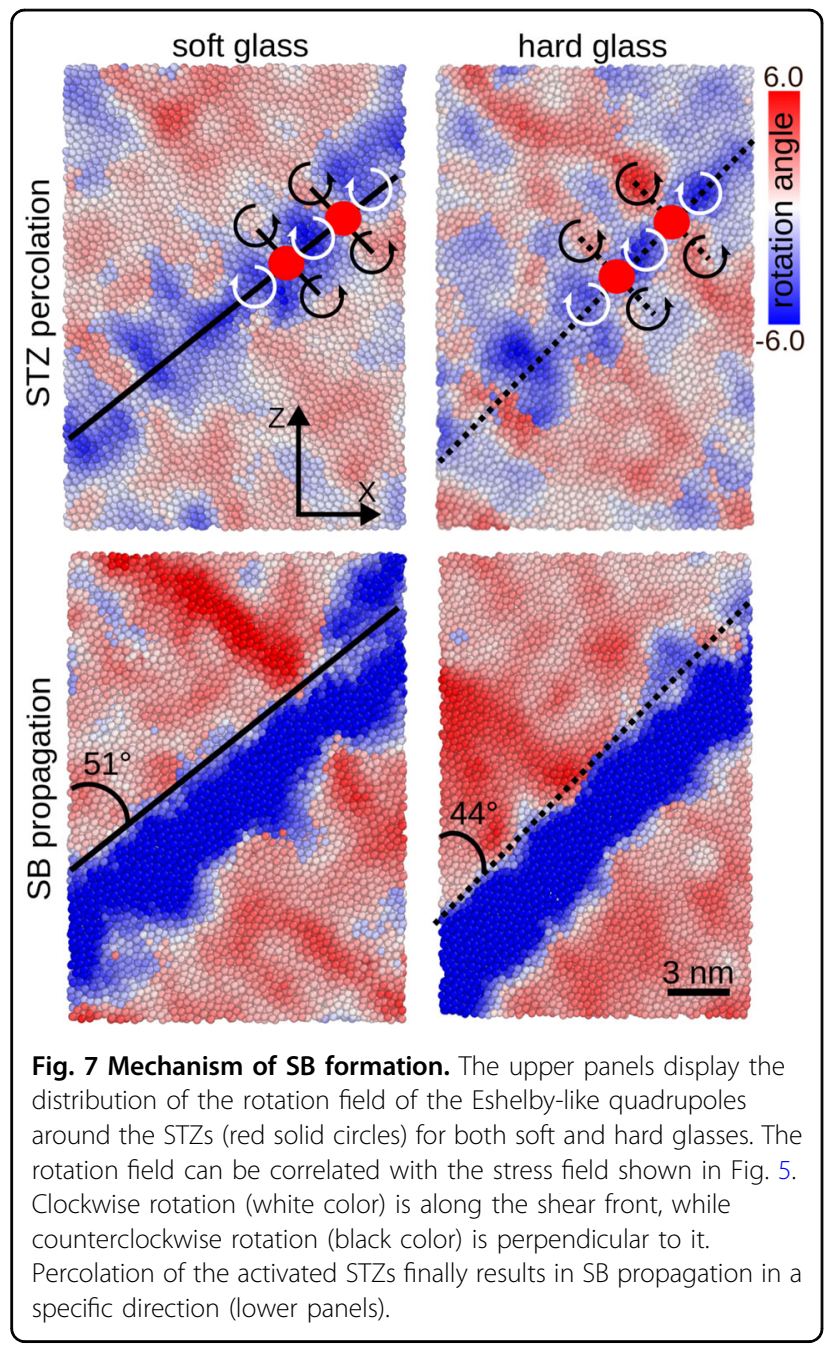

experiment can be found in Supplementary Note 2. An increased degree of heterogeneity induces a complex stress field around STZs, transforming the uniaxial stress field into a multiaxial complex stress field. This slows the STZ percolation process and thus the formation of critical SBs, which delays the shear failure of MGs. Therefore, we believe that there is an essential, in-depth link between structural/elastic heterogeneities, the dynamics of the STZ percolation process, the progressive formation of $\mathrm{SBs}$, and enhanced strain to failure.

\section{Conclusions}

We performed tension experiments and in situ highenergy synchrotron XRD investigations on a monolithic MG at cryogenic temperatures. Macroscopically, the overall density increases with decreasing temperature, causing a $43 \%$ increase in the yield strength. In addition, the changes in the atomic-level structural heterogeneity related to the increased nonaffine thermal strain of the
SRO and the MRO might control the STZ percolation process and consequently change the SB dynamics and directional angle. Based on Eshelby's inclusion analysis, we proposed a model to explain the STZ percolation mechanism and the strong variations in SB dynamics. With decreasing temperature, inhomogeneous fluctuations in the atomic packing structure induce a compression-overwhelmed stress field around the STZ. MD simulations revealed how variations in the stress field around the STZ affect the STZ percolation path and influence the shear-banding process. Furthermore, a high degree of heterogeneity slows the rate of STZ percolation and ultimately decelerates SB sliding. Therefore, stressstate modification on the microscale provides a feasible strategy for enhancing the shear stability and guiding the design of new ductile MGs. These findings can expand our understanding of the relationship between the atomic-scale structure and deformation mechanisms in MGs.

\begin{abstract}
Acknowledgements
The authors would like to acknowledge the financial support from the National Key Research and Development Program of China (No. 2017YFB0701902), the NSFC (grant nos. 51925103, 51761135125, 51671120, 51827801, and 51801027) and the 111 project (No. D16002). Parts of this research were carried out with the light source PETRA III (beamline P02.1) at DESY, a member of the Helmholtz Association (HGF). Additional support through the European Research Council under the ERC Advanced Grant INTELHYB (grant ERC-2013-ADG-340025) is gratefully acknowledged. D.S. acknowledges the financial support by the German Science Foundation (DFG) through grant SO 1518/1-1.
\end{abstract}

\section{Author details}

'Laboratory for Microstructures, Institute of Materials, Shanghai University, 200444 Shanghai, China. ${ }^{2}$ Erich Schmid Institute of Materials Science, Austrian Academy of Sciences, Jahnstraße 12, A-8700 Leoben, Austria. ${ }^{3}$ Technische Universität Darmstadt, Institut für Materialwissenschaft, Fachgebiet Materialmodellierung, Otto-Berndt-Straße 3, D-64287 Darmstadt, Germany. ${ }^{4}$ Institute of Physics, Chinese Academy of Science, 100190 Beijing, China. ${ }^{5}$ DESY Photon Science, Notkestr. 85, D-22607 Hamburg, Germany. ${ }^{6}$ Department of Materials Science, Chair of Materials Physics, Montanuniversität Leoben, Jahnstraße 12, A-8700 Leoben, Austria

\section{Author contributions}

G.W. and J.E. conceived the project. X.B. and G.W. designed and conducted the tensile experiments and structure analysis. J.B. carried out the in situ synchrotron diffraction measurements. B.S. contributed to the Eshelby inclusion analysis. D.Ş performed the MD simulations. X.B. and G.W. wrote the paper with support from all authors. All authors discussed and commented on the paper.

\section{Conflict of interest}

The authors declare that they have no conflict of interest.

\section{Publisher's note}

Springer Nature remains neutral with regard to jurisdictional claims in published maps and institutional affiliations.

Supplementary information is available for this paper at https://doi.org/ 10.1038/s41427-020-00241-4.

Received: 23 April 2020 Revised: 23 June 2020 Accepted: 19 July 2020. Published online: 11 September 2020 


\section{References}

1. Schuh, C. A., Hufnagel, T. C. \& Ramamurty, U. Mechanical behavior of amorphous alloys. Acta Mater. 55, 4067-4109 (2007).

2. Chen, M. W. A brief overview of bulk metallic glasses. NPG Asia Mater. 3, 82-90 (2011).

3. Ketov, S. V. et al. Rejuvenation of metallic glasses by non-affine thermal strain. Nature 524, 200-203 (2015).

4. Pan, J., Ivanov, Y. P., Zhou, W. H., Li, Y. \& Greer, A. L. Strain-hardening and suppression of shear-banding in rejuvenated bulk metallic glass. Nature $\mathbf{5 7 8}$, 559-562 (2020).

5. Bian, X. L. et al. Manipulation of free volumes in a metallic glass through Xeion irradiation. Acta Mater. 106, 66-77 (2016).

6. Ma, E. \& Ding, J. Tailoring structural inhomogeneities in metallic glasses to enable tensile ductility at room temperature. Mater. Today 19, 568-579 (2016).

7. Liu, Y. H., Wang, G., Wang, R. J., Pan, M. X. \& Wang, W. H. Super plastic bulk metallic glasses at room temperature. Science 315, 1385-1388 (2007).

8. Zhu, F., Song, S., Reddy, K. M., Hirata, A. \& Chen, M. W. Spatial heterogeneity as the structure feature for structure-property relationship of metallic glasses. Nat. Commun. 9, 1-7 (2018).

9. Ross, P. et al. Linking macroscopic rejuvenation to nano-elastic fluctuations in a metallic glass. Acta Mater. 138, 111-118 (2017).

10. Tsai, P., Kranjc, K. \& Flores, K. M. Hierarchical heterogeneity and an elastic microstructure observed in a metallic glass alloy. Acta Mater. 139, 11-20 (2017).

11. Liu, C. Y. \& Maaß, R. Elastic fluctuations and structural heterogeneities in metallic glasses. Adv. Funct. Mater. 28, 1800388 (2018).

12. Bian, $X$. L. et al. Controlling the distribution of structural heterogeneities in severely deformed metallic glass. Mater. Sci. Eng. A 752, 36-42 (2019).

13. Pauly, S., Gorantla, S., Wang, G., Kühn, U. \& Eckert, J. Transformation-mediated ductility in CuZr-based bulk metallic glasses. Nat. Mater. 9, 473-477 (2010).

14. Wu, Y., Xiao, Y., Chen, G., Liu, C. T. \& Lu, Z. P. Bulk metallic glass composites with transformation-mediated work-hardening and ductility. Adv. Mater. 22, 2770-2773 (2010).

15. Spaepen, F. A microscopic mechanism for steady state inhomogeneous flow in metallic glasses. Acta Metall. 25, 407-415 (1977).

16. Argon, A. S. Plastic deformation in metallic glasses. Acta Metall. 27, 47-58 (1979).

17. Schall, P., Weitz, D. A. \& Spaepen, F. Structural rearrangements that govern flow in colloidal glasses. Science 318, 1895-1899 (2007).

18. Hieronymus-Schmidt, V., Rösner, H., Wilde, G. \& Zaccone, A. Shear banding in metallic glasses described by alignments of Eshelby quadrupoles. Phys. Rev. $B$ 95, 134111 (2017)

19. Sun, B. A. et al. Transformation-mediated plasticity in CuZr based metallic glass composites: a quantitative mechanistic understanding. Int. J. Plast. 85, 34-51 (2016).

20. Eshelby, J. D. The determination of the elastic field of an ellipsoidal inclusion, and related problems. Proc. R. Soc. Lond. Ser. A 241, 376-396 (1957).

21. Ding, J., Patinet, S., Falk, M. L., Cheng, Y. \& Ma, E. Soft spots and their structural signature in a metallic glass. Proc. Natl. Acad. Sci. USA 111, 14052-14056 (2014).

22. Greer, A. L., Cheng, Y. Q. \& Ma, E. Shear bands in metallic glasses. Prog. Mater. Sci. 74, 71-132 (2013).

23. Egami, T. Atomic level stresses. Prog. Mater. Sci. 56, 637-653 (2011).

24. Bulatov, V. V. \& Argon, A. S. A stochastic model for continuum elasto-plastic behavior. I. Numerical approach and strain localization. Model. Simul. Mater. Sci. Eng. 2, 167-184 (1994).

25. Sopu, D., Stukowski, A., Stoica, M. \& Scudino, S. Atomic-level processes of shear band nucleation in metallic glasses. Phys. Rev. Lett. 119, 195503 (2017).

26. Scudino, S. \& Şopu, D. Strain distribution across an individual shear band in real and simulated metallic glasses. Nano Lett. 18, 1221-1227 (2018).

27. Şopu, D., Scudino, S., Bian, X. L., Gammer, C. \& Eckert, J. Atomic-scale origin of shear band multiplication in heterogeneous metallic glasses. Scr. Mater. 178 57-61 (2020).
28. Hammersley, A. P., Svensson, S. O., Hanfland, M., Fitch, A. N. \& Hausermann, D. Two-dimensional detector software: from real detector to idealised image or two-theta scan. High Press. Res. 14, 235-248 (1996).

29. Juhás, P., Davis, T., Farrow, C. L. \& Billinge, S. J. PDFgetX3: a rapid and highly automatable program for processing powder diffraction data into total scattering pair distribution functions. J. Appl. Crystallogr. 46, 560-566 (2013).

30. Plimpton, S. Fast parallel algorithms for short-range molecular dynamics. J. Comput. Phys. 117, 1-19 (1995).

31. Mendelev, M. I., Sordelet, D. J. \& Kramer, M. J. Using atomistic computer simulations to analyze $x$-ray diffraction data from metallic glasses. J. Appl. Phys. 102, 043501 (2007)

32. Maaß, R., Klaumünzer, D., Preiß, E. I., Derlet, P. M. \& Löffler, J. F. Single shearband plasticity in a bulk metallic glass at cryogenic temperatures. Scr. Mater. 66, 231-234 (2012).

33. Bian, X. L. et al. Atomic origin for rejuvenation of a Zr-based metallic glass at cryogenic temperature. J. Alloy. Compd. 718, 254-259 (2017).

34. Bian, $X$. et al. Cryogenic-temperature-induced structural transformation of a metallic glass. Mater. Res. Lett. 5, 284-291 (2017).

35. Wang, W. H. The elastic properties, elastic models and elastic perspectives of metallic glasses. Prog. Mater. Sci. 57, 487-656 (2012).

36. Ding, J. \& Ma, E. Computational modeling sheds light on structura evolution in metallic glasses and supercooled liquids. npj Comput. Mater. $\mathbf{3}$ 1-12 (2017)

37. Sukhomlinov, S. V. \& Müser, M. H. Determination of accurate, mean bond lengths from radial distribution functions. J. Chem. Phys. 146, 024506 (2017).

38. Gangopadhyay, A. K. \& Kelton, K. F. A re-evaluation of thermal expansion measurements of metallic liquids and glasses from $\mathrm{x}$-ray scattering experiments. J. Chem. Phys. 148, 204509 (2018).

39. Hufnagel, T. C., Ott, R. T. \& Almer, J. Structural aspects of elastic deformation of a metallic glass. Phys. Rev. B 73, 064204 (2006).

40. Shahabi, H. S. et al. Structural aspects of elasto-plastic deformation of a Zr-based bulk metallic glass under uniaxial compression. Acta Mater. 95, 30-36 (2015).

41. Poulsen, H. F., Wert, J. A., Neuefeind, J., Honkimäki, V. \& Daymond, M. Measuring strain distributions in amorphous materials. Nat. Mater. 4, 33-36 (2005).

42. Küchemann, S. \& Maaß, R. Gamma relaxation in bulk metallic glasses. Scr. Mater. 137, 5-8 (2017)

43. Zhang, Z. F., He, G., Eckert, J. \& Schultz, L. Fracture mechanisms in bulk metallic glassy materials. Phys. Rev. Lett. 91, 045505 (2003).

44. Jiang, M. Q. et al. Cryogenic-temperature-induced transition from shear to dilatational failure in metallic glasses. Acta Mater. 77, 248-257 (2014).

45. Sun, B. A., Pauly, S., Hu, J., Wang, W. H., Kühn, U. \& Eckert, J. Origin of intermittent plastic flow and instability of shear band sliding in bulk metallic glasses. Phys. Rev. Lett. 110, 225501 (2013).

46. Johnson, W. L. \& Samwer, K. A universal criterion for plastic yielding of metallic glasses with a (T/Tg)2/3 temperature dependence. Phys. Rev. Lett. 95, 195501 (2005).

47. Daub, E. G., Klaumünzer, D. \& Löffler, J. F. Effective temperature dynamics of shear bands in metallic glasses. Phys. Rev. E 90, 062405 (2014).

48. Langer, J. S. Dynamics of shear-transformation zones in amorphous plasticity: formulation in terms of an effective disorder temperature. Phys. Rev. E 70 041502 (2004).

49. Haxton, T. K. \& Liu, A. J. Activated dynamics and effective temperature in a steady state sheared glass. Phys. Rev. Lett. 99, 195701 (2007).

50. Wright, W. J., Samale, M. W., Hufnagel, T. C., LeBlanc, M. M. \& Florando, J. N. Studies of shear band velocity using spatially and temporally resolved measurements of strain during quasistatic compression of a bulk metallic glass. Acta Mater. 57, 4639-4648 (2009).

51. Maaß, R., Klaumünzer, D. \& Löffler, J. F. Propagation dynamics of individual shear bands during inhomogeneous flow in a Zr-based bulk metallic glass. Acta Mater. 59, 3205-3213 (2011)

52. Wang, G. et al. Nanoscale periodic morphologies on the fracture surface of brittle metallic glasses. Phys. Rev. Lett. 98, 235501 (2007).

53. Qu, R. T., Liu, Z. Q., Wang, G. \& Zhang, Z. F. Progressive shear band propagation in metallic glasses under compression. Acta Mater. 91, 19-33 (2015). 